\title{
Immunotherapy in Breast Cancer: the New Frontier
}

\author{
Zishuo I. $\mathrm{Hu}^{1} \cdot$ Heather L. McArthur ${ }^{2}$ \\ Published online: 16 April 2018 \\ (C) The Author(s) 2018
}

\begin{abstract}
Purpose of review This review summarizes current immunotherapies in breast cancer, with an emphasis on immune checkpoint inhibitors and vaccines.

Recent findings Combination immunotherapy with checkpoint inhibitors and cytotoxic therapies have shown promising results. Active clinical trials are ongoing in both early stage and metastatic settings for triple negative, HER2+, and hormone-positive breast cancer patients.

Summary Ongoing challenges remain in defining biomarkers that predict response to immunotherapy, determining the optimal combination immunotherapies, and enhancing the immunogenicity of breast cancer subtypes.
\end{abstract}

Keywords Breast cancer $\cdot$ Immunotherapy $\cdot$ Checkpoint inhibitors $\cdot$ Vaccines

\section{Introduction}

Immunotherapy has recently proven a promising therapeutic strategy for both hematological and solid malignancies. Although most breast cancers are not inherently immunogenic, recent efforts employing checkpoint inhibitors either as monotherapy or in combination with local or systemic strategies have yielded promising results. Herein, we discuss immunotherapy strategies under investigation for breast cancer, with a focus on immune checkpoint inhibitors and cancer vaccines. We also review future challenges and growing areas of research in the field of breast cancer immunotherapy.

\section{Immune Checkpoint Inhibitors}

Immune checkpoint inhibitors enable $\mathrm{T}$ cell activation by agonizing costimulatory signals or antagonizing co-inhibitory

This article is part of the Topical Collection on Translational Research

Heather L. McArthur

Heather.McArthur@cshs.org

1 Mount Sinai Health System, Icahn School of Medicine, New York, NY, USA

2 Department of Medicine, Breast Oncology, Samuel Oschin Comprehensive Cancer Institute, Cedars-Sinai Medical Center, 8700 Beverly Blvd, 1S35, Los Angeles, CA 90048, USA signals. Specifically, T cell inhibition is interrupted with the administration of antibodies that bind to cytotoxic $\mathrm{T}$ lymphocyte antigen 4 (CTLA-4) or programmed death-1 or its ligand (PD-L1), thereby permitting robust and sustained $\mathrm{T}$ cell responses. T cell activation is initiated with the presentation of antigens to the major histocompatibility complex (MHC) molecule on the surface of antigen presenting cells (APCs). After binding of the $\mathrm{T}$ cell receptor (TCR) to the MHC-antigen complex, the B7 protein on the APC binds to the T cell costimulatory molecule $\mathrm{CD} 28$ to promote $\mathrm{T}$ cell activation and survival. Inhibition of $\mathrm{T}$ activation is mediated by the subsequent upregulation of CTLA-4 on T cells, which competes with $\mathrm{CD} 28$ to bind B7. Further control of T cells response in peripheral tissue is regulated by the expression of PD-1 on activated T cells. The binding of PD- 1 to its ligand PD-L1 induces an inhibitory signal that limits T cell proliferation and survival [1-3].

\section{CTLA-4 Inhibitors}

One of the first clinical trials to administer checkpoint inhibitor therapy in breast cancer was a phase 1 study using the monoclonal fully human CTLA-4 antibody, tremelimumab, in combination with exemestane in 26 post-menopausal women with metastatic hormone-positive or hormone-responsive breast cancer [4]. The best reported overall response (ORR) was durable, stable disease in 11 of the 26 patients $(42 \%)$. The majority of the treatment-related adverse events (AE) were 
mild to moderate, with the most common being diarrhea $(46 \%)$ and pruritus (42\%). Furthermore, 7 (27\%) had grade 3 AEs, with one serious AE and no grade 4 AEs. Notably, treatment was also associated with an increase in the percent of activated CD4+ and CD8+ T cells as measured by inducible costimulatory (ICOS) expression and marked increase in the ratio of peripheral activated $T$ cells to suppressive regulatory $T$ cells. More recently, CTLA4-blockade has been explored in combination with local strategies such as tumor freezing, or cryoablation, in the curative-intent setting (see below) $[5,6]$.

\section{PD-1/PD-L1 Inhibitors}

PD-1/PD-L1 Inhibitors as Monotherapy for Metastatic TNBC One of the first reports of PD-1/PD-L1 inhibition in breast cancer was a phase $1 \mathrm{~b}$ trial of the anti-PD-1 antibody pembrolizumab in 32 women with PD-L1-"positive" metastatic triple negative breast cancer (mTNBC) (KEYNOTE-012) [7]. In this study, PD-L1 positivity was defined as PD-L1 expression in the stroma or in $\geq 1 \%$ of tumor cells. The overall response rate was $18.5 \%$ in 27 evaluable patients, and the median time to response was 17.9 weeks (range, 7.3 to 32.4 weeks). Median overall survival (OS) was 10.2 months, and the 12 -month OS rate was $41.1 \%$. A total of $15.6 \%$ of the patients had at least one grade 3 to $5 \mathrm{AE}$, with the most common AE being arthralgia (18.8\%) and fatigue (18.8\%). Of the five responders, one had a complete response (CR), four had partial responses (PRs), and three have had long-lasting benefit from pembrolizumab. At the time of publication, median duration of response had not yet been reached.

In a phase $1 \mathrm{~b}$ study, the anti-PD-L1 antibody, atezolizumab, was evaluated in women with PDL1-"positive" metastatic TNBC [8]. The PD-L1 tumor-infiltrating immune cell (IC) status was defined by the percentage of PD-L1-positive ICs: IC 0/1 <5\% or negative and IC $2 / 3$ ( $\geq$ $5 \%)$ or positive. Of the response evaluable population $(N=$ $21)$, the ORR was $19 \%$. Of the safety evaluable population $(N=54), 11 \%$ of the patients had at least one grade 3 to $5 \mathrm{AE}$. This trial was later expanded to include patients regardless of PD-L1 status [9]. In the expansion, the ORR for the 112 evaluable patients was $10 \%$ overall. Patients with high PDL1-expressing tumors had higher ORRs compared to patients with low PD-L1 expression (13 versus 5\%, respectively). The ORR was $26 \%$ for patients treated with atezolizumab in the first-line versus $4 \%$ in the second-line setting with a 21 -month median duration of response which is remarkable for this generally poor prognosis population.

In the JAVELIN study, the PD-L1-directed antibody avelumab demonstrated modest responses across breast cancer subtypes with an ORR of $8.6 \%$ in the TNBC cohort unselected for PD-L1 expression [10]. Notably, there was no impact on response by various tumor PD-L1 cut-offs.
In KEYNOTE-086, pembrolizumab was administered as either salvage treatment for metastatic TNBC patients regardless of PD-L1 expression (cohort A) or as first-line therapy for patients with metastatic PD-L1+ TNBC (cohort B) [11, 12]. The ORR was $4.7 \%$ in the 170 previously treated patients (cohort A), with no significant difference observed by PDL1 expression. Median OS was 8.9 months. The ORR for the 52 previously untreated patients with PD-L1-positive TNBC (cohort B) was $23 \%$. Thus, $23-26 \%$ of women with TNBC respond to PD-1 or PD-L1 blockade in the first-line setting. However, because very modest responses are observed with monotherapy in the second-line setting and beyond, combination strategies are needed if the majority of immunologically "cold" tumors are to be converted into immune-responsive tumors.

\section{PD-1/PD-L1 Inhibitors in Combination with Chemotherapy for} mTNBC The combination of chemotherapy with PD-1/PD-L1targeted therapies has shown promising results in mTNBC. For example, in a phase $1 \mathrm{~b}$ study of 32 patients with evaluable mTNBC, the combination of atezolizumab and nab-paclitaxel resulted in an ORR of $38 \%$, with similar response rates in the first versus third line and beyond (46 versus $40 \%$, respectively) [13]. The ORR by PD-L1 expression was $30 \%$ for IC0, $36 \%$ for IC1/2/3, and $46 \%$ for unknown expression, respectively. A phase 3 multicenter, randomized, double-blind placebo-controlled trial (IMpassion130, NCT02425891) is ongoing to assess nab-paclitaxel with or without atezolizumab as first-line treatment in mTNBC.

In phase 1 study of eribulin with pembrolizumab in mTNBC, an ORR of $34 \%$ was reported overall, with an ORR of $41 \%$ in an interim analysis of 17 patients treated in the first-line setting versus $27 \%$ in the 2 nd and 3 rd line settings [14]. Consistent with the nab-paclitaxel with atezolizumab experience, no significant differences in responses were observed by PD-L1 expression in the eribulin with pembrolizumab study. Moreover, across studies, when responses occur, they tend to be durable.

PD-1/PD-L1 Inhibitors in Combination with Chemotherapy for Early Stage TNBC Given the encouraging results observed with chemotherapy/checkpoint blockade combinations in the mTNBC setting, there is considerable interest in applying these strategies with curative intent. The I-SPY2 trial has an adaptive randomization design that allows for expeditious evaluation of drugs in the pre-operative setting to determine whether they are likely to be successful in a randomized study. In a recent report from I-SPY2, paclitaxel was administered with or without pembrolizumab followed by four cycles of conventional doxorubicin with cyclophosphamide (AC) in women with early stage HER2-normal disease in the preoperative setting [15]. When pembrolizumab was added to standard chemotherapy, the estimated pathologic complete 
response rate (pCR) was approximately $20 \%$ in the control arm versus $60 \%$ in the pembrolizumab-containing arm for the subset of women with TNBC. Although the estimated pCR rate in the control arm is less than expected for preoperative chemotherapy with a taxane and anthracycline in TNBC, the magnitude of the difference across the arms was striking. However, the addition of pembrolizumab to the chemotherapy backbone added to the toxicity of the regimen with a rate of adrenal insufficiency of 8.7 versus $0 \%$ for the pembrolizumab versus control arms, respectively. In KEYNOTE-173, a phase 1 b trial of neoadjuvant pembrolizumab with anthracycline and taxane-based chemotherapy in TNBC, a pCR rate of 80 versus $50 \%$ was achieved with or without the addition of carboplatin [16]. However, the improvement in pCR with the addition of carboplatin was associated with an $80 \%$ grade $3 / 4$ neutropenia rate. Thus, close safety monitoring is critical as these combination strategies are being explored in the curative-intent setting. There are currently several large randomized studies planned or underway. For example, in KEYNOTE-522, women with stage II/ III TNBC are being randomized at diagnosis to receive neoadjuvant chemotherapy (including an anthracycline, taxane, and carboplatinum) with or without pembrolizumab (NCT03036488) [17]. In Southwest Oncology Group (SWOG) 1418, patients with residual TNBC after neoadjuvant chemotherapy are randomized to a year of pembrolizumab versus observation (NCT02954874). And in IMpassion030, women with stage II/III TNBC will be randomized to anthracycline and taxane-based adjuvant chemotherapy with or without a year of adjuvant atezolizumab (NCT03197935). It is hoped that these chemotherapycheckpoint blockade combination strategies will improve cure rates and potentially permit de-escalation of cytotoxic backbones.

\section{PD-1/PD-L1 Inhibitors in Hormone Receptor-Positive, HER2-Negative Disease}

KEYNOTE-028 was a phase $1 \mathrm{~b}$ multicohort study of pembrolizumab for PD-L1-positive advanced tumors [18]. In this study, PD-L1 positivity was again defined as PD-L1 expression in the stroma or in $\geq 1 \%$ of tumor cells. Among the 25 patients with estrogen-positive/HER2-negative metastatic breast cancer treated on study, an ORR of $12 \%$ was reported with three PRs with only $16 \%$ of patients experiencing a grade 3 or grade $4 \mathrm{AE}$. More modest response rates were observed in the phase $1 \mathrm{~b}$ JAVELIN study, wherein 72 women with hormone receptor-positive, HER2-negative disease were treated with avelumab irrespective of PD-L1 expression [10]. In this subset, an ORR of $2.8 \%$ was observed. However, in the ISPY2 study, wherein pre-operative chemotherapy was administered with or without pembrolizumab in a curative-intent population, the estimated pCR rate was 34 versus $13 \%$, respectively, in women with hormone receptor-positive/ HER2-normal breast cancer [15]. Thus, although hormone receptor-positive disease may not be innately sensitive to checkpoint blockade monotherapy, chemotherapy combinations - particularly when administered earlier in the course of disease - may be particularly effective.

\section{PD-1/PD-L1 Inhibitors in HER2-Positive Disease}

Trastuzumab has been shown to have immune-mediated mechanisms of action [19, 20], and pre-clinical studies have demonstrated synergy with checkpoint blockade in combination with HER2-directed therapy [21]. To date, however, there is a paucity of data exploring checkpoint blockade in this subset. In the phase $1 \mathrm{~b}$ JAVELIN study, one response $(3.8 \%)$ was observed among the 26 women with metastatic HER2-positive disease treated with avelumab [10].

In PANACEA, 12 women with HER2-positive, PD-L1negative, and 40 women with HER2-positive, PD-L1positive disease with prior progression on trastuzumab received concurrent trastuzumab with pembrolizumab in the phase II portion of the study [22]. The best overall response was $15 \%$ in the PD-L1-positive and $0 \%$ in the PD-L1-negative cohorts. Several studies exploring rational combinations of HER2-directed therapy with checkpoint blockade in the palliative- and curative-intent setting are planned or underway.

\section{Combinations of Local Strategies (Cryoablation or Radiation) with Checkpoint Blockade}

In a pilot study, the CTLA-4-directed antibody, ipilimumab, versus primary tumor cryoablation versus the combination was explored prior to mastectomy in 19 women with early stage breast cancer [5]. Specifically, 7 women received tumor cryoablation alone, 6 women received a single-dose of ipilimumab alone, and 6 women received the combination of tumor cryoablation and ipilimumab. Cryoablation and ipilimumab (at $10 \mathrm{mg} / \mathrm{kg}$ ) were safe alone and in combination, with no treatment-associated Grade 3/4 AEs reported. The combination was also associated with increased levels of activated and proliferating CD4+ and CD8+ T cells, and posttreatment proliferative T-effector cells. Furthermore, ipilimumab alone increased intratumoral $\mathrm{T}$ cell density over time, whereas cryoablation with or without ipilimumab resulted in clonal expansion of $\mathrm{T}$ cells as demonstrated by deep sequencing of T cell receptor DNA [6]. A follow-up pilot study (NCT02833233) evaluated the combination of cryoablation, ipilimumab, and the PD-1 antibody nivolumab in the pre-operative setting, and the results of that effort are expected to directly inform a large randomized study.

Because radiation is immunogenic and can optimize antigen presentation, combinations of radiation with checkpoint blockade are being explored. A study of standard-of-care 
brain radiation with tremelimumab-mediated CTLA4 blockade for women with breast cancer brain metastases conferred non-CNS disease control in $10 \%(2 / 20)$ of women with HER2-normal disease [23]. However, tremelimumab coadministered with trastuzumab and brain radiation in women with HER2-positive disease conferred non-CNS disease control in 33\% (2/6). In a study combining non-CNS radiation with pembrolizumab in women with TNBC, 3 of $9(33 \%)$ evaluable women at 12 weeks had a partial response outside of the radiation field [24]. A similar study combining radiation with pembrolizumab in hormone receptor-positive metastatic breast cancer is underway (NCT03051672).

The TONIC trial (NCT02499367) is a phase 2 randomized trial that aimed to explore the issue of priming for checkpoint blockade. Specifically, nivolumab was administered after induction therapy with radiation $(3 \times 8$ Gy of radiation to one metastatic lesion), low-dose chemotherapy (doxorubicin weekly, cyclophosphamide daily, or cisplatin weekly for 2 weeks), or no induction therapy in 50 patients with mTNBC [25]. Initial results reported an ORR of $10 \%$ in patients treated with RT, $45 \%$ with doxorubicin, $11 \%$ with cyclophosphamide, $33 \%$ with cisplatin, and $11 \%$ with no induction. Thus, this small study underscores the importance of dose, schedule, and sequence whenever checkpoint blockade is combined with systemic or local strategies.

\section{Cancer Vaccines in Breast Cancer}

A number of cancer vaccine strategies have been tested in breast cancer, including monovalent vaccines, polyvalent vaccines, and cellular vaccines. Monovalent vaccines provide a single tumor-associated antigen (TAA) target for the immune system. Polyvalent peptide vaccines provide multiple TAA targets, and cellular vaccines are ex vivo modified tumor cells or APCs.

\section{Monovalent Vaccines}

Targets used in monovalent vaccines for breast cancer include HER2, mucin 1 (MUC1), and carcinoembryonic antigen (CEA). The E75 peptide vaccine, nelipepimut-S, is derived from the extracellular domain of the HER2 protein. In clinical trials, it has been tested with granulocyte-macrophage colonystimulating factor (GM-CSF) and has been found to be safe in phase I and II clinical trials. GP2 is an MHC Class I peptide derived from the transmembrane domain of the HER2 protein. The GP2 peptide combined with GM-CSF found the vaccine to be safe and capable of generating a HER2-specific T cell response in early phase trials. A subsequent phase II study with 180 patients was performed with an intention-to-treat analysis performed at a median follow-up of 34 months [26]. The analysis showed an $88 \%$ estimated 5-year disease-free survival (DFS) rate in vaccinated patients versus $81 \%$ in GM-CSF-only patients $(p=0.43)$. Treatment analysis, which excluded recurrences during the primary vaccination series and secondary malignancies, showed $100 \%$ DFS in vaccinated HER2-positive patients compared with $89 \%$ in GM-CSFtreated only patients $(p=0.08)$.

MUC1 is a glycoprotein normally expressed on the cell surface of epithelial cells in the breast, stomach, lung, and urinary tracts. It has been found to be overexpressed in all breast cancers and its epitopes CA 15-3 and CA 27-29 serve as breast tumor markers. The MUC1 epitope, sialyl-Tn (STn), was conjugated with the keyhole limpet hemocyanin (KLH) carrier protein, to form the cancer vaccine Theratope. Despite promising pre-clinical and phase II clinical trials, a multicenter, randomized, phase III trial that consisted of 1028 patients with metastatic breast cancer found no significant differences in median time to progression (TTP) or overall survival time between patients who received the vaccine compared with patients who received the KLH carrier protein alone [27].

\section{Polyvalent Vaccines}

PANVAC (pancreatic vaccine) is a recombinant poxviral vaccine expressing MUC1, CEA, and three T cell costimulatory molecules. A pilot trial that included 12 metastatic breast cancer patients resulted in four patients with stable disease and one patient with a complete response to therapy [28]. A subsequent phase II trial combining PANVAC with docetaxel revealed a median PFS of 7.9 months in patients treated with docetaxel and PANVAC compared to 3.9 months in patients treated with docetaxel alone $(p=0.09)$ [29].

\section{Cellular Vaccines}

The GVAX breast vaccine is an allogeneic, HER2-targeted GM-CSF-secreting cellular vaccine. It was evaluated in combination with a range of a low-dose cyclophosphamide and doxorubicin in 28 patients with metastatic breast cancer [30]. Low-dose cyclophosphamide was able to selectively induce the apoptosis of regulatory $\mathrm{T}$ cells compared to effector $\mathrm{T}$ cells [31]. The vaccine was also given in combination with trastuzumab and low-dose cyclophosphamide to 20 patients with metastatic HER2+ breast cancer [32]. The authors reported an increase in immune response markers to the vaccine, including polyfunctional HER2-specific CD8+ T cells and delayed type hypersensitivity.

\section{Conclusions}

The clinical experience with immunotherapy for the treatment of breast cancer has grown rapidly in recent years. Checkpoint blockade has been most effective as monotherapy in the first- 
line mTNBC setting and in combination with chemotherapy in pre-treated mTNBC. However, checkpoint blockade is particularly promising in the curative-intent setting across tumor types. Checkpoint blockade in breast cancer has been associated with tolerable safety profiles, and among responders, shown to have durable responses that compare favorably to standard chemotherapy.

One ongoing challenge is defining the patient population that would most benefit from immunotherapy with checkpoint blockade and/or vaccines. For example, trials of checkpoint blockade have demonstrated mixed results with respect to PDL1 expression as a predictive marker in breast cancer. Tumorinfiltrating lymphocytes have been more robust as a predictive biomarker across multiple studies of multiple agents; however, reliable and reproducible predictive biomarkers are still needed. Mutational burden, CD8+ T cell density, and oncogenic mutations are all markers currently under scrutiny and evaluation [33]. Combination therapy may serve to act as an equalizing agent for patients with lower expression levels of PD-L1, promoting tumor immunogenicity and increasing response rates. Further research into identifying predictive markers in the setting of a growing number of combinatorial therapy trials is of particular clinical relevance both for checkpoint blockade and vaccine-based strategies. It is certain that as more combinations with immunotherapy are explored, the successful identification of rational therapeutic partners will be paramount.

\section{Compliance with Ethical Standards}

Conflict of Interest The authors declare that they have no competing interests.

Human and Animal Rights and Informed Consent This article does not contain any studies with human or animal subjects performed by any of the authors.

Open Access This article is distributed under the terms of the Creative Commons Attribution 4.0 International License (http:// creativecommons.org/licenses/by/4.0/), which permits unrestricted use, distribution, and reproduction in any medium, provided you give appropriate credit to the original author(s) and the source, provide a link to the Creative Commons license, and indicate if changes were made.

\section{References}

1. O'Shea JJ, Paul WE. Mechanisms underlying lineage commitment and plasticity of helper CD4+ T cells. Science. 2010;327:1098102. https://doi.org/10.1126/science.1178334.

2. Leach DR, Krummel MF, Allison JP. Enhancement of antitumor immunity by CTLA-4 blockade. Science. 1996;271:1734-6. https://doi.org/10.1126/science.271.5256.1734.

3. Topalian SL, Drake CG, Pardoll DM. Targeting the PD-1/B7H1(PD-L1) pathway to activate anti-tumor immunity. Curr Opin
Immunol. 2012;24:207-12. https://doi.org/10.1016/j.coi.2011.12. 009 .

4. Vonderheide RH, LoRusso PM, Khalil M, et al. Tremelimumab in combination with exemestane in patients with advanced breast cancer and treatment-associated modulation of inducible costimulator expression on patient T cells. Clin Cancer Res: Off J Am Assoc Cancer Res. 2010;16:3485-94. https://doi.org/10.1158/1078-0432. CCR-10-0505.

5. McArthur HL, Diab A, Page DB, et al. A pilot study of preoperative single-dose ipilimumab and/or cryoablation in women with earlystage breast cancer with comprehensive immune profiling. Clin Cancer Res: Off J Am Assoc Cancer Res. 2016;22:5729-37. https://doi.org/10.1158/1078-0432.CCR-16-0190.

6. Page DB, Yuan J, Redmond D, Wen YH, Durack JC, Emerson R, et al. Deep sequencing of T-cell receptor DNA as a biomarker of clonally expanded TILs in breast cancer after immunotherapy. Cancer Immunol Res. 2016;4:835-44. https://doi.org/10.1158/ 2326-6066.CIR-16-0013.

7. Nanda R, Chow LQ, Dees EC, et al. Pembrolizumab in patients with advanced triple-negative breast cancer: phase Ib KEYNOTE012 study. J Clin Oncol Off J Am Soc Clin Oncol. 2016;34:2460-7. https://doi.org/10.1200/JCO.2015.64.8931.

8. Emens LA, Braiteh FS, Cassier P, Delord JP, Eder JP, Fasso M, et al. Abstract 2859: inhibition of PD-L1 by MPDL3280A leads to clinical activity in patients with metastatic triple-negative breast cancer (TNBC). Cancer Res. 2015;75:2859. https://doi.org/10.1158/15387445.AM2015-2859.

9. Schmid P, Cruz C, Braiteh FS, Eder JP, Tolaney S, Kuter I, et al. Abstract 2986: atezolizumab in metastatic TNBC (mTNBC): longterm clinical outcomes and biomarker analyses. Cancer Res. 2017;77:2986. https://doi.org/10.1158/1538-7445.AM2017-2986.

10. Dirix LY, Takacs I, Jerusalem G, et al. Avelumab, an anti-PD-L1 antibody, in patients with locally advanced or metastatic breast cancer: a phase $1 \mathrm{~b}$ JAVELIN Solid Tumor study. Breast Cancer Res Treat 2017.

11. Adams S, Loi S, Toppmeyer D, et al. Phase 2 study of pembrolizumab as first-line therapy for PD-L1-positive metastatic triple-negative breast cancer (mTNBC): preliminary data from KEYNOTE-086 cohort B. J Clin Oncol. 2017;35:1088.

12. Adams S, Schmid P, Rugo HS, et al. Phase 2 study of pembrolizumab (pembro) monotherapy for previously treated metastatic triple-negative breast cancer (mTNBC): KEYNOTE-086 cohort A. J Clin Oncol. 2017;35:1008.

13. Adams S, Diamond JR, Hamilton EP, et al. Phase Ib trial of atezolizumab in combination with nab-paclitaxel in patients with metastatic triple-negative breast cancer (mTNBC). 2016 ASCO Annual Meeting 2016. J Clin Oncol, 2016;34:(suppl; abstr 1009).

14. Tolaney S, Savulsky C, Aktan G, et al. Phase $1 \mathrm{~b} / 2$ study to evaluate eribulin mesylate in combination with pembrolizumab in patients with metastatic triple-negative breast cancer. Eur J Cancer. 2017;72: S16. https://doi.org/10.1016/S0959-8049(17)30131-4.

15. Nanda R, Liu MC, Yau C, et al. Pembrolizumab plus standard neoadjuvant therapy for high-risk breast cancer $(\mathrm{BC})$ : results from I-SPY 2. J Clin Oncol. 2017;35:506.

16. Schmid P, Park YH, Muñoz-Couselo E, et al. Pembrolizumab (pembro) + chemotherapy (chemo) as neoadjuvant treatment for triple negative breast cancer (TNBC): preliminary results from KEYNOTE-173. J Clin Oncol. 2017;35:556.

17. Schmid P, Cortes Castan J, Bergh J, et al. 233TiPKEYNOTE-522: Phase III study of pembrolizumab (pembro) + chemotherapy (chemo) vs placebo + chemo as neoadjuvant followed by pembro vs placebo as adjuvant therapy for triple-negative breast cancer (TNBC). Ann Oncol. 2017;28:mdx364.015-mdx364.015. https:// doi.org/10.1111/dom.13279.

18. Rugo H, Delord J-P, Im S-A, et al. Abstract S5-07: preliminary efficacy and safety of pembrolizumab (MK-3475) in patients with 
PD-L1-positive, estrogen receptor-positive (ER+)/HER2-negative advanced breast cancer enrolled in KEYNOTE-028. Cancer Res. 2016;76:S5-07-S5. https://doi.org/10.1093/annonc/mdy012.

19. Clynes RA, Towers TL, Presta LG, Ravetch JV. Inhibitory Fc receptors modulate in vivo cytotoxicity against tumor targets. Nat Med. 2000;6:443-6. https://doi.org/10.1038/74704.

20. Park S, Jiang Z, Mortenson ED, Deng L, Radkevich-Brown O, Yang X, et al. The therapeutic effect of anti-HER2/neu antibody depends on both innate and adaptive immunity. Cancer Cell. 2010;18:160-70. https://doi.org/10.1016/j.ccr.2010.06.014.

21. Stagg J, Loi S, Divisekera U, Ngiow SF, Duret H, Yagita H, et al. Anti-ErbB-2 mAb therapy requires type I and II interferons and synergizes with anti-PD-1 or anti-CD137 mAb therapy. Proc Natl Acad Sci U S A. 2011;108:7142-7.

22. Loi S, Giobbe-Hurder A, Gombos A et al. Phase Ib/II study evaluating safety and efficacy of pembrolizumab and trastuzumab in patients with trastuzumab-resistant HER2-positive metastatic breast cancer: results from the PANACEA (IBCSG 45-13/BIG 4-13/ KEYNOTE-014) study. 2017 SABCS. San Antonio, Texas 2017.

23. McArthur H, Beal K, Halpenny D, Henrich M, Modi S, Patil S, et al. Abstract 4705: CTLA4 blockade with HER2-directed therapy $(\mathrm{H})$ yields clinical benefit in women undergoing radiation therapy (RT) for HER2-positive (HER2+) breast cancer brain metastases (BCBM). Cancer Res. 2017;77:4705. https://doi.org/10.1158/ 1538-7445.AM2017-4705.

24. McArthur HL, Barker CA, Gucalp A et al. A single-arm, phase II study assessing the efficacy of pembrolizumab (pembro) plus radiotherapy (RT) in metastatic triple negative breast cancer (mTNBC). 2018 ASCO-SITC Clinical Immuno-Oncology Symposium 2017.

25. Kok M, Horlings HM, van de Vijver K, et al. LBA14 Adaptive phase II randomized non-comparative trial of nivolumab after induction treatment in triple negative breast cancer: TONIC-trial. Ann Oncol. 2017;28:mdx440.006-mdx440.006. https://doi.org/10.1186/ s12961-018-0294-0.

26. Mittendorf EA, Ardavanis A, Litton JK, Shumway NM, Hale DF, Murray JL, et al. Primary analysis of a prospective, randomized, single-blinded phase II trial evaluating the HER2 peptide GP2 vaccine in breast cancer patients to prevent recurrence. Oncotarget. 2016;7:66192-201. https://doi.org/10.18632/ oncotarget.11751.

27. Miles D, Roche H, Martin M, Perren TJ, Cameron DA, Glaspy J, et al. Phase III multicenter clinical trial of the sialyl-TN (STn)keyhole limpet hemocyanin (KLH) vaccine for metastatic breast cancer. Oncologist. 2011;16:1092-100. https://doi.org/10.1634/ theoncologist.2010-0307.

28. Mohebtash M, Tsang KY, Madan RA, Huen NY, Poole DJ, Jochems C, et al. A pilot study of MUC-1/CEA/TRICOM poxviral-based vaccine in patients with metastatic breast and ovarian cancer. Clin Cancer Res: Off J Am Assoc Cancer Res. 2011;17: 7164-73. https://doi.org/10.1158/1078-0432.CCR-11-0649.

29. Heery CR, Ibrahim NK, Arlen PM, Mohebtash M, Murray JL, Koenig K, et al. Docetaxel alone or in combination with a therapeutic cancer vaccine (PANVAC) in patients with metastatic breast cancer: a randomized clinical trial. JAMA Oncol. 2015;1:1087-95. https://doi.org/10.1001/jamaoncol.2015.2736.

30. Emens LA, Asquith JM, Leatherman JM, Kobrin BJ, Petrik S, Laiko M, et al. Timed sequential treatment with cyclophosphamide, doxorubicin, and an allogeneic granulocyte-macrophage colonystimulating factor-secreting breast tumor vaccine: a chemotherapy dose-ranging factorial study of safety and immune activation. J Clin Oncol Off J Am Soc Clin Oncol. 2009;27:5911-8. https://doi.org/ 10.1200/JCO.2009.23.3494.

31. Chen G, Leatherman JM, Sunay ME, Emens LA. Abstract 4749: cyclophosphamide induces dose dependent apoptosis of CD4+ FoxP3+ regulatory $\mathrm{T}$ cells relative to $\mathrm{CD} 4+\mathrm{FoxP} 3$ - effector T cells in breast cancer patients. Cancer Res. 2013;73:4749.

32. Chen G, Gupta R, Petrik S, Laiko M, Leatherman JM, Asquith JM, et al. A feasibility study of cyclophosphamide, trastuzumab, and an allogeneic GM-CSF-secreting breast tumor vaccine for HER2+ metastatic breast cancer. Cancer Immunol Res. 2014;2:949-61. https://doi.org/10.1158/2326-6066.CIR-14-0058.

33. Topalian SL, Taube JM, Anders RA, Pardoll DM. Mechanismdriven biomarkers to guide immune checkpoint blockade in cancer therapy. Nat Rev Cancer. 2016;16:275-87. https://doi.org/10.1038/ nrc. 2016.36 . 